\title{
INFLUENCIA DE LA ESTABILIDAD DE PRECIOS Y EL DESEMPEÑO DEL SISTEMA FINANCIERO EN EL CRECIMIENTO ECONÓMICO DE MÉXICO, 1994-2013*
}

\author{
ISELA ELIZABETH TÉLLEZ-LEÓN** \\ FRANCISCO VENEGAS-MARTÍNEZ***
}

Recibido: 13 de junio de 2012 • Aprobado: 05 de octubre de 2013

\section{RESUMEN}

En este artículo se examina la influencia del sistema financiero en el crecimiento económico y la relación de dicho sistema con la estabilidad de precios y otras variables macroeconómicas, entre 1994 y 2013. Para ello, se estima un modelo de vectores auto-regresivos, se examinan las funciones de impulsorespuesta, se analiza la descomposición de la varianza y se efectúan pruebas de causalidad de Granger. Se encuentra evidencia de que en México la estabilidad de precios y el sistema financiero han influido en el crecimiento económico.

\section{PALABRAS CLAVE}

Estabilidad de precios, crecimiento económico, sistema financiero, vectores auto regresivos.

\section{CLASIFICACIÓN JEL}

E31, F43, G20, C13

\section{CONTENIDO}

Introducción; 1. Hechos estilizados sobre sistema financiero y el crecimiento económico; 2. Aplicación empírica para México 1994-2013, especificación del modelo; 3. Estimaciones econométricas y análisis de los resultados; 4. Conclusiones; Bibliografía; Anexos.

* Artículo de investigación, motivado por las recurrentes opiniones que consideran a la política monetaria de México un medio para promover el crecimiento económico, cuando por mandato constitucional el Banco de México tiene por objetivo prioritario la estabilidad de precios. En este sentido, se aporta luz en el tema para observar que si Banxico se enfoca en su objetivo prioritario permite una condición necesaria para el crecimiento económico, la cual es la estabilidad de precios; se considera que complementando con otras políticas económicas se podría apoyar el crecimiento económico, por ejemplo a través del impulso del sistema financiero que posibilite la inversión productiva. El periodo de elaboración del proyecto fue de agosto de 2012 a junio de 2013. Los autores pertenecen al Sistema Nacional de Investigadores de México.

** Licenciada en Economía, UAM, México D. F. Magíster en Economía, CIDE, México D. F. Doctora en Ciencias Económicas del Instituto Politécnico Nacional, Sección de Estudios de Posgrado de la Escuela Superior de Economía, México D. F. Correo electrónico: tellezelizabeth_2015@yahoo.com.

*** Licenciado en Matemáticas, UNAM, México D. F. Magíster en Economía, ITAM, México D. F. Doctor en Economía de University of Washington State. Postdoctorado en Economía por la University of Oxford. Profesor-Investigador de la Escuela Superior de Economía, ESE-IPN. Correo electrónico: fvenegas1111@ yahoo.com.mx. 


\title{
INFLUENCE OF PRICE STABILITY AND PERFORMANCE OF THE FINANCIAL SYSTEM IN ECONOMIC GORWTH IN MEXICO 1994-2013
}

\begin{abstract}
This paper analyses the influence of the financial system in economic growth and the relation that system has in the price stability and other macroeconomic variables between 1994 and 2013. For this purpose an Autoregressive Vector model is estimated, the impulse-response functions are studied, the decomposition of the variance is analyzed and a Ganger causality test is performed. Evidence is found that in Mexico the stability of prices and the financial system has influenced economic growth.
\end{abstract}

\section{KEYWORDS}

Price stability, economic growth, financial system, autoregressive vectors.

\section{JEL CLASSIFICATION}

E31, F43, G20, C13

\section{CONTENT}

Introduction; 1. Stylized facts about the financial system and economic growth; 2. Empiric application for Mexico 1994-2003, Model specification; 3. Econometric estimations and result analysis; 4. Conclusions; Bibliography; Attachments.

\section{INFLUÊNCIA DA ESTABILIDADE DE PREÇOS E 0 DESEMPENHO DO SISTEMA FINANCEIRO NO CRESCIMENTO ECONÔMICO DO MÉXICO, 1994-2013}

\section{RESUMO}

Neste artigo se examina a influência do sistema financeiro no crescimento econômico e a relação de dito sistema com a estabilidade de preços e outras variáveis macroeconômicas, entre 1994 e 2013. Para isso, estima-se um modelo de Vectores Auto-regressivos, examina-se as funções de impulso-resposta, analisase a decomposição da variância e se efetua provas de causalidade de Granger. Encontra-se evidência de que no México a estabilidade de preços e o sistema financeiro influíram no crescimento econômico.

\section{PALAVRAS CHAVES}

Estabilidade de preços, crescimento econômico, sistema financeiro, vectores auto regressivos.

\section{CLASSIFICAÇÃO JEL}

E31, F43, G20, C13

\section{CONTEÚDO}

Introdução; 1. Fatos estilizados sobre sistema financeiro e o crescimento econômico; 2. Aplicação empírica para México 1994-2013, especificação do modelo; 3. Estimações econométricas e análises dos resultados;

4. Conclusões; Bibliografia; Anexos. 
Influencia de la estabilidad de precios y el desempeño del sistema financiero en el crecimiento económico de México, 1994-2013

\section{INTRODUCCIÓN}

Este artículo de investigación ha sido motivado por las recurrentes opiniones que consideran a la política monetaria de México un medio para promover el crecimiento económico, cuando por mandato constitucional el Banco de México (Banxico) tiene por objetivo prioritario la estabilidad de precios. En este sentido, se aporta luz en el tema para observar que si Banxico se enfoca en su objetivo prioritario permite una condición necesaria para el crecimiento económico, la cual es la estabilidad de precios; se considera que complementado con otras políticas económicas se podría apoyar el crecimiento económico, por ejemplo, a través del impulso del sistema financiero que posibilite la inversión productiva.

En este documento se analiza la influencia de la estabilidad de precios y el sistema financiero, como condiciones necesarias para impulsar la inversión productiva, las cuales propician un escenario sano para el crecimiento económico sostenido. Se realiza un análisis empírico sobre la relación de dichas variables en México de 1994 a 2013. En este sentido, esta investigación presenta un tema innovador y que conjuga dos estudios, porque se pretende: 1) obtener evidencia empírica que acepte o rechace la hipótesis de que un sistema financiero eficiente proporciona crecimiento económico en un marco de estabilidad de precios, para el caso de México de 19942013; y, 2) observar la causalidad de las variables para esta muestra: PIB real, la razón inversión a producto, el financiamiento de la banca comercial al sector no bancario, el índice de precios y cotizaciones (IPC), los recursos totales de las entidades de ahorro y crédito popular. Las anteriores tres variables se consideran representantes del sistema financiero. Además, como variables exógenas se agregan: la tasa de interés, la inflación y el tipo de cambio real.

Esta investigación acentúa la importancia del desarrollo de un sistema financiero y la estabilidad de precios como escenario clave para el crecimiento económico sostenido. Si bien existen diversas teorías, el consenso sugiere que la inversión productiva requiere financiamiento, el cual es una condición fundamental para el crecimiento económico; en palabras de Wray (2002): "el financiamiento es una precondición para que cualquier producción tome lugar en realidad". Este trabajo sostiene la necesidad de contar con un sistema financiero eficiente y orientado al financiamiento del sector productivo.

En términos de estabilidad de precios, esta es necesaria porque evita distorsiones asociadas con la inflación, lo cual facilita la creación de proyectos de inversión. Para una estimación del efecto de la inflación sobre el crecimiento de largo plazo véase Barro (1996) y para una evaluación del efecto de la estabilidad en México en comparación con Asia del Este, véase De Gregorio y Lee (2004). 
Lo anterior implica que debe revisarse por qué México ha presentado un crecimiento económico moderado. La respuesta de algunos economistas se halla en la curva de Phillips aumentada, que muestra la relación inversa entre crecimiento e inflación. Sin embargo, otros economistas consideran que puede coexistir un ambiente que proteja el poder adquisitivo y, a su vez, impulse el crecimiento, esto se puede lograr si se consolida un sistema financiero (véase Levine, 1997), que proporcione crédito al sector productivo y se acompañe de otras medidas de política económica.

La conjunción de políticas económicas es necesaria, si las autoridades desean un crecimiento económico sostenido con estabilidad de precios, porque no es posible que un instrumento de política ataque dos objetivos económicos (crecimiento y control de la inflación). Esto ya ha sido demostrado por la vasta literatura de la curva de Phillips, en especial, puede verse en el modelo de la curva de Phillips aumentada con expectativas, el cual proporciona una completa explicación del trade-off entre la tasa de inflación y tasa de desempleo (además véase el planteamiento de Chari (1998) en términos de la postura neoclásica'.

En otros enfoques surgen fuertes críticas hacia la ortodoxia neoclásica, por la confección de políticas económicas con altas tasas de interés, un tipo de cambio sobrevaluado, extrema confianza en el desarrollo exterior y el establecimiento de metas de inflación, como la postura del latino-americanismo de Bresser-Pereira (2007).

Lo anterior ilustra posturas dentro del marco teórico sobre el tema, lo indiscutible es que detrás de estas posturas existe una discusión metodológica captada por Taylor (2000), por lo cual, es fundamental revisar el modelo macroeconómico que se ha seguido en México desde 1994 para entender un poco más las causas del bajo crecimiento y si en verdad no pueden coexistir estabilidad de precios y crecimiento económico.

Debido a la dependencia de México con Estados Unidos (EE. UU.), la política económica que ha regido dicho país afecta, sin duda, a México. Por esto es importante dedicar unas líneas al análisis del desempeño de los EE. UU.: hasta antes de 1980 en EE. UU., la política económica se había comprometido con el pleno empleo, por lo cual los salarios crecieron en función de la productividad, con el llamado Tratado de Detroit, lo que implicaba una fuerte demanda agregada que llevaba al pleno empleo. No obstante, después de 1980, la política económica se retiró del compromiso con el pleno empleo, y en su lugar las autoridades establecieron un nuevo modelo de

\footnotetext{
Otro ejemplo en Golosov y Lucas (2003).
} 
Influencia de la estabilidad de precios y el desempeño del sistema financiero en el crecimiento económico de México, 1994-2013

crecimiento neoliberal con bajos niveles de inflación, como motor del crecimiento de la demanda agregada sin fomentar el incremento de los salarios.

Así, el modelo neoliberal propuesto mostró más interés por la inflación que por el pleno empleo; a través de los postulados del Consenso de Washington, que fueron impulsados por el Banco Mundial y el Fondo Monetario Internacional (FMI), se extiende la agenda neoliberal mundial, al sugerir que todos los países adoptarían una política económica similar. De esta forma se abandonó la visión de Keynes y los postulados del modelo IS-LM-AS², como marco teórico, el cual había tenido efectos en la recuperación de los EE. UU. después de la crisis de 1929, ya que como lo expresa Romer (2000) tales modelos fueron elaborados para el corto plazo.

Una vez que se han revisado algunos de los enfoques prevalecientes, se concluye que todo radica en el plazo sobre el cual se elabora el análisis. En este sentido, se encuentra en la literatura un aspecto fundamental: la distinción entre el corto, el mediano y el largo plazo, ya que, en función de los períodos de tiempo, alguna de las teorías disponibles podría apegarse más a la realidad, premisa que comparte Solow (2000), quien llega a la conclusión reconciliadora de seguir un modelo híbrido, porque logra combinar el instrumental de los enfoques a fin de proporcionar mejores recomendaciones en materia de política económica, de acuerdo con el contexto de la economía.

Con base en la discusión teórica anterior y la evidencia empírica, se considera que es necesario un nuevo modelo de referencia, por ejemplo, el modelo híbrido de Solow (2000) con una adecuada regulación financiera. En este trabajo se analiza la influencia de la estabilidad de precios y el sistema financiero en el crecimiento económico. El canal por el que se considera el impacto es la inversión productiva.

Para algunos autores es debatible la prioridad de la política monetaria del Banco de México, dado el trade-off de corto plazo entre estabilidad de precios y crecimiento económico3. La política monetaria ha sido efectiva en su objetivo de mantener la estabilidad de precios; aunque se ha observado un crecimiento moderado, esto no es consecuencia de la política monetaria emprendida por México, ya que el crecimiento económico no es su objetivo. El crecimiento modesto de México fue resultado de la falta de reformas estructurales, las cuales ya se han emprendido en este sexenio;

2 Modelo IS-LM-AS: Model Investment Saving-Liquidity Preference Money Supply-Aggregate Supply.

3 El instrumento de política monetaria empleado en un inicio por el Banco de México (Banxico) fue el régimen de saldos acumulados (largos y cortos). A partir del 10 de abril de 2003 se sustituyó el régimen de saldos acumulados por uno de saldos diarios. En este momento, el instrumento de política monetaria es similar al empleado por la FED (Sistema de Reserva Federal de los Estados Unidos), una tasa de interés objetivo. 
estas prometen mucho, una vez que se aprueben las leyes secundarias que permitirán operar dichas reformas. Por lo tanto, se considera que estas y las debidas políticas económicas permitirán en el mediano plazo un despegue del crecimiento económico de México. Esta investigación muestra cómo una de las alternativas fuertes para impulsar el crecimiento económico es un eficaz sistema financiero, esto con la debida regulación que permita la asignación eficiente de recursos.

La principal contribución del presente trabajo consiste en estudiar la influencia del sistema financiero, la estabilidad de precios y la macroeconómica que posibiliten las condiciones de inversión como uno de los motores del crecimiento económico. Se encuentra evidencia de cómo durante el período 1994:01-2013:04, en México, la estabilidad ha influido en el crecimiento económico y que el sistema financiero tiene un peso fuerte en este. Para ello, se estima un modelo de vector auto-regresivos (VAR) que permite analizar la coexistencia de la estabilidad de precios con el crecimiento económico y estudiar la influencia del sistema financiero en el crecimiento económico.

La presente investigación está organizada de la siguiente forma: en la primera sección se establecen los hechos estilizados del sistema financiero mexicano y el crecimiento económico en México; en la segunda sección se presenta la especificación del modelo para la aplicación empírica de México 1994-2013, a través de un modelo VAR; por último, en la tercera sección se presentan las estimaciones econométricas y se efectúa el análisis de los resultados. Finalmente, se presentan las conclusiones y se proporciona una serie de recomendaciones en materia de política económica para impulsar el crecimiento económico en condiciones de estabilidad de precios y de firmeza macroeconómica.

\section{HECHOS ESTILIZADOS SOBRE SISTEMA FINANCIERO Y EL CRECIMIENTO ECONÓMICO}

El sistema financiero mexicano, en la segunda mitad del siglo XX, experimentó un marco regulatorio que desalentó la competencia entre intermediarios financieros y generó distorsiones en la asignación de recursos, lo que afectó las actividades productivas y con ello al crecimiento económico del país.

En las décadas de 1970 y 1980, cuando se presentaron eventos de inestabilidad macroeconómica, el sistema financiero estaba estancado y los recursos financieros que circulaban se desplazaron hacia el financiamiento del alto y creciente déficit fiscal; esto desincentivó la inversión (lo que se conoce como el efecto crowding out). Por esta razón, las posteriores políticas de regulación del sistema financiero se caracterizaron por una fuerte intervención, en términos de asignación de fondos y fijación de precios, lo cual reducía el margen de operación al sistema financiero, 
Influencia de la estabilidad de precios y el desempeño del sistema financiero en el crecimiento económico de México, 1994-2013

lo que generó su ineficiencia en este período. Sin embargo, para finales de los ochentas, el estancamiento del sistema financiero mexicano debía ser combatido, si se quería impulsar la inversión. Por ello, en 1989 se dio un proceso de desregulación que se profundizó en 1990. En los últimos años, la regulación emprendida por Banxico pretende generar expectativas favorables para el sano desarrollo del sistema financiero mexicano.

Dicho fortalecimiento de la regulación en México surgió a raíz de la crisis financiera en EE. UU., entre 2007-2009, ya que esta mostró que la falta de regulación y la excesiva expansión del crédito hacia sectores improductivos impactan en el desempeño de la economía ${ }^{4}$. Diversos estudios de las crisis derivan una conclusión fundamental (por ejemplo, véanse Caballero, Farhi y Gourinchas, 2008): las crisis internacionales tienen causas en función del enfoque teórico que sigue el modelo económico del país.

Esto puede notarse en las causas de la gran depresión ${ }^{5}$, las cuales son diferentes a las de la reciente crisis, pero en ambas se han observado repercusiones fuertes en EE. UU. y al resto de los países, resultado del marco teórico seguido por las autoridades. La reciente crisis ha puesto en evidencia la vulnerabilidad del sistema financiero de EE. UU, por la falta de regulación. Por ello, es importante replantear el modelo de crecimiento económico y regular el sistema financiero para aprovecharlo como motor del crecimiento económico en el país. En resumen, el problema de la reciente crisis financiera no tiene como raíz un problema de productividad laboral y eficiencia (causas atribuidas a la crisis de 1929 en EE. UU.), sino de desregulación del sistema financiero, ya que la ausencia de una regulación financiera ha desencadenado graves problemas en el mecanismo de financiamiento para la inversión productiva.

Por ello, es fundamental comprender la crisis financiera que se gestó en 2007, la cual entra en la hipótesis de la inestabilidad financiera de Minsky (1982)6 ${ }^{6}$, para

$4 \quad$ Si se agrega el contexto global de una crisis, entonces las conexiones psicológicas se incrementan por la euforia o pesimismo de los inversionistas. A medida que el boom especulativo continúa, las tasas de interés, la velocidad de circulación y los precios aumentan. En algún momento, la información privilegiada para pocos influye en la decisión de retirar ganancias y vender, los precios de los activos comienzan a descender con consecuencias desastrosas para algunos prestatarios que no podrán solventar sus obligaciones. Los especuladores se dan cuenta, poco a poco o de repente, que el mercado ya no incrementará los rendimientos, lo cual es una señal para los inversionistas de retirarse, entonces los activos financieros de largo plazo pueden emprender la fuga, si el país tiene una alta dependencia de éstos, esto ocasionará un estancamiento del crédito, no habrá liquidez ni financiamiento hasta que se recupere la confianza en la economía del país, esta situación se podría convertir en un laberinto sin salida.

5 Véanse Chari, Kehoe y McGrattan (2002).

6 En este sentido, el ciclo básico de una crisis por expansión del crédito fue retratada por Minsky (1982), quien concede gran importancia al papel de la estructura de la deuda como causa de dificultades 
así conocer las implicaciones de política económica y regulación que un sistema financiero debe seguir, con la finalidad de evitar las crisis financieras, el apalancamiento excesivo, los excesos de riesgos, los requisitos inadecuados al capital, entre otros. La señal específica que precipita la crisis puede ser el fracaso de un banco o una empresa, la revelación de una estafa o desfalco financiero, o una caída en los precios de los activos, ingrediente principal para la especulación y las quiebras, lo que abrirá paso al pánico, caso en el cual se requiere del prestamista de última instancia, lo que aún es una cuestión debatible. Del mismo modo, es debatible la intervención del prestamista de última instancia cuando este pretende salvar a un agente económico que no fue prudente. Lo anterior podría evitarse si se establecen medidas regulatorias adecuadas y se reestructura el modelo económico de un país.

En consecuencia, la regulación financiera es necesaria para asegurar la estabilidad económica, fomentar la inversión productiva y motivar el crecimiento económico. En palabras de Summers (2008) "los excesos financieros son el único problema para que el crecimiento vuelva a ser normal". Este argumento va en consonancia con los defensores de Minsky (1982), como Kregel (2008), Whalen (2007) y Wray (2008). Así, la hipótesis de la inestabilidad financiera parece dar una explicación completa a la reciente crisis y esta solo se afrontará con regulación financiera.

En estos años, la reforma del sector financiero es solo uno de los pasos; también es necesario reestructurar el modelo de crecimiento tecnológico a fin de restablecer el vínculo entre salario y productividad, lo cual se prevé considerado en las reformas estructurales que emprendió México en 2013. Esto implicará una restructuración económica, la activación del mercado interno y la restauración de la meta de pleno empleo, la cual se espera mejore la condición de vida de las familias que no pueden mantener su consumo debido al estancamiento de los salarios y a la desigualdad del ingreso.

financieras y en especial a la deuda contraída para apalancar la adquisición de activos especulativos. El trabajo de Minsky (1982) sobre la fragilidad del sistema financiero ha resurgido con gran fuerza en la literatura especializada. Minsky (1982) propone una teoría psicológica basada en el ciclo económico con agentes optimistas, la cual inicia con manifestaciones optimistas por los valores y la disposición para asumir riesgos, dadas las expectativas futuras. Esta psicología optimista afecta tanto a los prestatarios como a los prestamistas. El ciclo continúa con el financiamiento de la cobertura, cuando se espera que los ingresos de los prestatarios sean suficientes para pagar el capital y los intereses del préstamo. Después se pasa a la fase de la especulación financiera, cuando los ingresos sólo cubren los intereses. Por último, el ciclo finaliza con finanzas Ponzi, cuando los ingresos son insuficientes para cubrir los pagos de intereses y afecta a los prestatarios, porque confían en las ganancias de capital para cumplir sus obligaciones convirtiéndose en un círculo vicioso, lo cual genera desconfianza, esto agudiza un factor negativo en las decisiones de inversión como lo había planteado Akerlof (1970). 


\section{APLICACIÓN EMPÍRICA PARA MÉXICO 1994-2013, ESPECIFICACIÓN DEL MODELO}

El sistema financiero de México está formado por el sistema bancario, casas de bolsa, afianzadoras, arrendadoras, aseguradoras, administradoras de los fondos de inversión y demás instituciones que intermedian entre quienes ahorran y quienes solicitan préstamos. Las referidas instituciones financieras monitorean los riesgos de liquidez y crédito, con la finalidad de hacer más eficiente la asignación de recursos. Los encargados de regular el sistema financiero en México son: Banco de México (Banxico); Secretaría de Hacienda y Crédito Público (SHCP); Comisión Nacional Bancaria y de Valores (CNBV); Instituto para la Protección al Ahorro Bancario (IPAB); Comisión Nacional para la Protección y Defensa de los Usuarios de Servicios Financieros (Condusef); Comisión Nacional de Seguros y Fianzas (CNSF); Comisión Nacional del Sistema de Ahorro para el Retiro (Consar); y, la Comisión Nacional del Sistema de Ahorro para el Retiro (Consar).

En muchas ocasiones se emplean indistintamente los términos sistema financiero y mercado financiero, lo cual no es correcto; por ello es importante señalar que los mercados financieros son aquellos donde se intercambian activos, entre los que se encuentran: el mercado accionario (se tranzan acciones, las empresas pueden emitir sus acciones en la Bolsa Mexicana de Valores (BMV) ${ }^{7}$ a través de una casa de bolsa); el mercado de deuda (se negocian instrumentos de deuda del Gobierno o empresas), y el mercado cambiario (se negocian monedas extranjeras, que facilitan el comercio internacional), mientras que, como se mencionó antes, el sistema financiero está formado por instituciones que gestionan la asignación eficiente de recursos entre los ahorradores y los demandantes de crédito. En este contexto, es conveniente explicitar que se estudia el sistema financiero.

Con la anterior distinción, se considera el modelo teórico y empírico que se propone en esta investigación. Existe una extensa literatura que estima la relación entre el sistema financiero y el crecimiento económico como: Demirgüç-Kunt y Levine (2004), Demirguc-Kunt y Maksimovic (2002), Levine (2002), Beck y Levine (2002) y Beck, Levine y Demirgüç-Kunt (2009). En los anteriores estudios se estiman modelos panel que han sido fuertemente criticados en los últimos años, por la heterogeneidad de los países, la endogeneidad de las variables y porque no resuelven la causalidad de la relación en estudio. Por ejemplo, De Gregorio y Guidotti (1995) ya habían señalado que dividiendo la muestra de países, las correlaciones cambian, lo que indica que se debe tomar especial atención al agrupar a los países, y sugiere

\footnotetext{
El 11 de enero de 1999 la negociación accionaria se incorporó completamente a un sistema electrónico de negociación, el Sistema Electrónico de Negociación, Transacción, Registro y Asignación (BMVSENTRA Capitales), para agilizar las operaciones porque anteriormente los títulos se intercambiaban de forma física.
} 
analizar país por país si se estudia el tema en cuestión. En esta investigación se opta por analizar a México a través de modelos de vectores auto-regresivos (VAR), dado que no se encontró un modelo similar aplicado al caso de México. Si bien, Carvajal y Zuleta (1997) estiman un VAR para el caso de Colombia, solo lo emplean para probar la hipótesis de causalidad.

\subsection{Especificación teórica del modelo}

Arestis y Demetriades (1997), Jung (1986), Christopoulos y Tsionas (2004), entre otros, han estudiado el tema bajo el enfoque individual, empleando el análisis de series de tiempo, por lo que se estudia la influencia del sistema financiero, la estabilidad de precios y macroeconómica como condiciones de inversión que permiten crecimiento económico a través de un modelo VAR, empleado, por ejemplo, en Arestis y Demetriades (1997).

Se retoman los fundamentos de Christopoulos y Tsionas (2004) para especificar la variable dependiente, como el PIB real y los regresores del modelo; se considera la incorporación de medidas de financiamiento (en nuestro estudio se emplea el IPC, el financiamiento otorgado por los bancos y por otras entidades no bancarias), la inflación, la tasa de interés y el tipo de cambio real (TCR). En Christopoulos y Tsionas (2004) se emplea la contribución de la inversión al producto; sin embargo, para tener una medida ponderada por el tamaño de la economía se emplea la inversión como parte del producto siguiendo a King y Levine (1993), ya que su principal conclusión es que el valor de los indicadores de desarrollo económico ayudan a predecir el valor promedio de la tasa de crecimiento económico. Para considerar la estabilidad macroeconómica se emplea una proxy, el tipo de cambio real (TCR). La representación del modelo VAR general es:

$$
y_{t}=\Pi_{1} y_{t-1}+\Pi_{2} y_{t-2}+\ldots+\Pi_{p} y_{t-p}+\Psi x_{t}+\varepsilon_{t}
$$

Donde $y_{t}$ es un vector de $\mathrm{k}$ variables endógenas, es un vector de variables exógenas, $\Pi$ y $\Psi$ son matrices de coeficientes y $\varepsilon_{t}$ es un vector de innovaciones.

\subsection{Estadística descriptiva de los datos}

La definición de las variables bajo estudio se proporciona a continuación: PIB real (Y) como una medida del crecimiento económico; el Índice de Precios y Cotizaciones de la Bolsa Mexicana de Valores (IPC); el financiamiento total otorgado por la Banca Comercial al sector no bancario (FIN _ BC); los recursos totales de las Entidades de Ahorro y Crédito Popular (EsyCrpop _ rectot); la razón inversión a producto (IAY); 
Influencia de la estabilidad de precios y el desempeño del sistema financiero en el crecimiento económico de México, 1994-2013

el Índice Nacional de Precios al Consumidor (INPC); el tipo de cambio real (TCR), y la tasa de interés de los $\mathrm{CETES}^{8}$ a 28 días (R).

Los datos fueron obtenidos de la Organización para la Cooperación y el Desarrollo Económico (OCDE) y de Banxico, con periodicidad trimestral de 1994:012013:049 . En el anexo A se presentan las gráficas en niveles de las series ajustadas por estacionalidad, en todas ellas se observa una tendencia y cierta variabilidad. Se puede observar la estadística descriptiva de las series en el cuadro 1.

Cuadro 1. Estadísticas de las series

\begin{tabular}{|l|c|c|c|c|c|c|c|c|}
\cline { 2 - 9 } \multicolumn{1}{c|}{} & Y & IPC & FIN_BC & $\begin{array}{c}\text { ESYCRPOP } \\
\text { RECTOT }\end{array}$ & IAY & R & INPC & TCR \\
\hline Promedio & $2,700,000,000,000$ & $16,402.9$ & $1,650,000,000$ & $26,200,000,000$ & 20.0 & 13.0 & 0.7 & 79.9 \\
\hline Mediana & $2,640,000,000,000$ & $9,127.3$ & $1,330,000,000$ & $10,900,000,000$ & 20.2 & 7.6 & 0.8 & 77.6 \\
\hline Máximo & $3,360,000,000,000$ & $43,837.0$ & $3,540,000,000$ & $108,000,000,000$ & 23.5 & 61.5 & 1.1 & 126.2 \\
\hline Mínimo & $1,960,000,000,000$ & $1,801.6$ & $610,000,000$ & $2,070,000,000$ & 14.3 & 3.2 & 0.2 & 57.4 \\
\hline Dev. Std. & $395,000,000,000$ & $13,770.9$ & $819,000,000$ & $30,100,000,000$ & 2.2 & 11.6 & 0.3 & 12.9 \\
\hline
\end{tabular}

Fuente: elaborada con base en datos de OCDE y Banxico

Con base en las gráficas y la estadística descriptiva de las series, se realizarán transformaciones a las series con la finalidad de estimar el modelo VAR con series estacionarias; para verificar el orden de integración de las series se realizan pruebas de raíces unitarias, que se muestran en el cuadro 2.

Cuadro 2. Pruebas de raíces unitarias

\begin{tabular}{|l|c|c|c|c|c|c|c|c|}
\cline { 2 - 9 } \multicolumn{1}{c|}{} & Y & IAY & IPC & FIN_BC & $\begin{array}{c}\text { ESYCRPOP } \\
\text { RECTOT }\end{array}$ & R & INPC & TCR \\
\hline Niveles & RU & RU & RU & RU & RU & NRU & RU & RU \\
\hline Logaritmo & RU & RU & RU & RU & RU & - & NRU & RU \\
\hline $\begin{array}{l}\text { Diferencia } \\
\text { del log }\end{array}$ & NRU & NRU & NRU & NRU & NRU & - & NRU & NRU \\
\hline
\end{tabular}

Nota: Pruebas realizadas tipo Dickey-Fuller Aumentado, bajo el criterio de Schwarz, con máximo 11 rezagos. $\mathrm{H}_{0}$ : La serie presenta raíz unitaria. Se rechaza la hipótesis nula al 95 por ciento de confianza.

RU: La serie presenta raíz unitaria. NRU: La serie no presenta raíz unitaria.

Fuente: elaborada con base en datos de OCDE y Banxico

8 Los CETES (Certificados de la Tesorería) son títulos de deuda pública emitidos por el Gobierno federal de México desde finales de la década de 1970.

9 Si el lector lo desea puede solicitar a los autores la base de datos que se empleó para la estimación de los modelos, favor de escribir un correo electrónico a itellez@banxico.org,mx. 
A continuación se estima un modelo VAR, para analizar las funciones de impulso respuesta y la descomposición de varianza de las variables antes mencionadas. Esto para obtener evidencia empírica que acepte o rechace la hipótesis de que un sistema financiero eficiente posibilita la inversión productiva, y con ella se impulsa el crecimiento económico, en un marco de estabilidad de precios y macroeconómica, para el caso de México de 1994-2013. Finalmente, se analiza la causalidad en el sentido de Granger de las series estudiadas para desarrollar el segundo objetivo de esta investigación.

Para seleccionar el rezago apropiado del modelo VAR se realiza la prueba de rezagos estándar; los resultados se presentan en el cuadro 3. Con base en el criterio de Schwarz (SC) y el de Hannan-Quinn (HQ), se toma la decisión de estimar un modelo VAR (1) y se estima un modelo VAR (2) con base en los criterios de Likelihood Ratio (LR), el error de predicción final (FPE) y Akaike (AIC). Lo anterior con la finalidad de evaluar entre los dos modelos y obtener el que mejor se ajuste a los datos, ya que con ese se realizará la inferencia estadística, en la siguiente sección.

Cuadro 3. Criterios de selección del rezago para el modelo VAR

\begin{tabular}{|c|c|c|c|c|c|c|}
\hline Criterio & 0 & 1 & 2 & 3 & 4 & 5 \\
\hline LR & NA & 98.04742 & $45.11476^{*}$ & 34.94534 & 15.35329 & 33.75253 \\
\hline FPE & $2.89 \mathrm{e}-15$ & $1.26 \mathrm{e}-15$ & $1.19 \mathrm{e}-15^{*}$ & $1.28 \mathrm{e}-15$ & $1.97 \mathrm{e}-15$ & $2.03 \mathrm{e}-15$ \\
\hline AIC & -19.29034 & -20.12309 & $-20.19932 *$ & -20.15902 & -19.79041 & -19.86479 \\
\hline SC & -18.66762 & $-18.72197 *$ & -18.0198 & -17.2011 & -16.05409 & -15.35007 \\
\hline HQ & -19.04193 & $-19.56416^{*}$ & -19.32989 & -18.97907 & -18.29994 & -18.06381 \\
\hline LogL & 733.7426 & 789.5542 & 817.3750 & 840.8837 & 852.2451 & 879.9972 \\
\hline
\end{tabular}

Variables Endógenas: DLY DLIAY DLIPC DLFIN _ BC DLESYCRPOP _ RECTOT.

Variables Exógenas: C R DLINPC DLTCR.

Muestra: 199401 201304.

* Indica el orden del rezago seleccionado por el Criterio. Cada prueba al 5\% de significancia. LR: Prueba estadística LR secuencial modificado. FPE: Error de predicción final. AIC: Criterio de Información de Akaike. SC: Criterio de Información de Schwarz. HQ: Criterio de Información de Hannan-Quinn.

Fuente: elaborada con base en datos de OCDE y Banxico. La DL antes de los nombres de las variables denota Diferencia del Logaritmo.

Una vez estimados el VAR (1) y el VAR (2), al comparar el criterio de información de cada modelo, el que mejor se ajustó fue el VAR (2), véase el Cuadro 4. 
Influencia de la estabilidad de precios y el desempeño del sistema financiero en el crecimiento económico de México, 1994-2013

Cuadro 4. Criterio de información de cada modelo VAR estimado

\begin{tabular}{|c|c|c|}
\hline Orden del VAR & AIC & SC \\
\hline 1 & 19.35765 & 17.99801 \\
\hline 2 & 19.17519 & 17.04446 \\
\hline
\end{tabular}

Fuente: elaborada con base en datos de OCDE y Banxico

Por lo tanto, en adelante el análisis se realizará sobre el VAR (2). La especificación de este se presenta en el anexo B. Se realizaron pruebas de diagnóstico al VAR (2), las cuales se presentan en el anexo F; estas sugieren que los residuos se distribuyen como una normal multivariada, no existe presencia de autocorrelación y las raíces del polinomio característico se encuentran dentro del círculo unitario.

\section{ESTIMACIONES ECONOMÉTRICAS Y ANÁLISIS DE LOS RESULTADOS}

Los coeficientes estimados por el modelo VAR que resultaron significativos al $95 \%$ de confianza, para el crecimiento económico son:

$$
\begin{gathered}
\operatorname{DLY}= \\
(0.0450)
\end{gathered}
$$

Los estimadores sugieren la interdependencia entre el sistema financiero y el crecimiento económico de México, como se muestra en la ecuación (2). Se puede inferir del VAR estimado que el crecimiento de la razón inversión a producto de un período anterior explica en promedio $12.7 \%$ de la tasa de crecimiento del producto real en el período corriente, mientras que, un incremento en la tasa de crecimiento del IPC explicaría en promedio $5.6 \%$ de la tasa de crecimiento del PIB real. El efecto contemporáneo se observa cuando una depreciación del TCR de $1 \%$ disminuye la tasa de crecimiento del PIB real en aproximadamente $7.0 \%$.

Observe que la tasa de crecimiento del financiamiento otorgado por la banca comercial al sector no bancario y la tasa de crecimiento de los recursos totales de las entidades de ahorro y crédito popular fueron estadísticamente no significativas, lo cual implica que al sistema financiero de México aún le falta profundizar o canalizar los recursos a la inversión productiva, para que se incentive la inversión que motiva el crecimiento económico. En tanto que, el primer rezago de la tasa de crecimiento del IPC y de la razón Inversión a PIB influye de forma positiva en el crecimiento del PIB real, esto da luz sobre las respuestas a las preguntas planteadas en esta investigación. La inversión productiva impacta en el crecimiento del PIB real de México 
significativamente, por lo que se requiere el impulso del sistema financiero que facilite la asignación de recursos entre prestatarios y prestamistas.

Las funciones de impulso respuesta muestran cómo un choque o innovación afecta a cada una de las variables; en especial, se observa que un choque de $1 \%$ en la tasa de crecimiento real de la economía es significativo en el primer y segundo períodos, $100 \%$ y $48.3 \%$, respectivamente. Un choque de $1 \%$ a la DLIAY tiene un impacto significativo sobre DLPIB en el segundo período; posteriormente el efecto se desvanece. Un choque positivo de $1 \%$ en el crecimiento del IPC impacta positivamente en 5.6 y $2.9 \%$ al DLPIB en el segundo y tercer períodos, en igual orden. El choque de $1 \%$ del DLFIN _ BC y DLESYCRPOP _ RECTOT no impacta a la tasa de crecimiento del PIB real, lo cual indica que estos sectores del sistema financiero (banca comercial y otros intermediarios no financieros) deberían otorgar más crédito a la inversión productiva para incentivar el crecimiento económico de México. Véanse las gráficas de las funciones de impulso-respuesta en el anexo C.

La descomposición de la varianza del DLPIB a diez períodos se muestra en el anexo D, con errores estándar derivados de la simulación Monte Carlo a 1000000 repeticiones. El porcentaje de la varianza del DLPIB debido a sus rezagos es fuerte, alrededor de $70 \%$ en la mayoría de los períodos, como se esperaba, mientras que la DLIAY está entre el $5 \%$ y $6 \%$ en casi todos los períodos, en tanto que, la variación de la tasa de crecimiento del IPC explica alrededor de 20 \% de la varianza en DLPIB. Las variaciones en la tasa de crecimiento del financiamiento de los bancos comerciales y de las entidades de ahorro y crédito popular son estadísticamente no significativas para el segundo período. Esto señala que en el período precedente dichas variables no tienen impacto sobre la variabilidad de la tasas de crecimiento del PIB real, pero potencialmente podrían influir en alrededor de $1 \%$.

A continuación se interpreta la casualidad en el sentido de Granger derivada del grupo de variables analizadas y del modelo VAR (2), (véase el anexo E). La causalidad en el sentido de Granger por pares indica que el IPC causa al PIB, el PIB causa a los recursos totales de las Entidades de Ahorro y Crédito popular, el IPC causa a la razón IAY, el financiamiento de la banca comercial al sector no bancario causa a la IAY (aunque existe esta relación, recuérdese que el impacto estimado fue no significativo, por lo que se recomienda impulsar el crédito de la banca comercial hacia los créditos productivos). El IPC causa al financiamiento de la banca comercial al sector no bancario, el IPC causa a los recursos totales de las Entidades de Ahorro y Crédito popular. Estas dos últimas relaciones muestran que el sistema financiero en México está bien articulado, y lo que se requiere es una mayor profundización dirigida a la inversión productiva. Existe una bi-direccionalidad entre el financiamiento de la 
Influencia de la estabilidad de precios y el desempeño del sistema financiero en el crecimiento económico de México, 1994-2013

banca comercial y los recursos totales de las entidades de ahorro y crédito popular. Finalmente, se observa que el INPC causa a los recursos totales de las entidades de ahorro y crédito popular; esto refuerza la hipótesis de que el Banco de México debe procurar mantener el poder adquisitivo de la moneda, su objetivo prioritario, porque si hubiera una inflación alta, esta influiría en los recursos que ahorran los individuos (con menores ingresos) en los intermediarios financieros, diferentes de la bolsa y la banca.

La causalidad estimada del modelo VAR (2) señala que la tasa de crecimiento del IPC causa fuertemente al crecimiento de la economía, al 95 \% de confianza, para la muestra dada. En resumen, se ha verificado que en México, para el periodo 1994:01-2013:04, la influencia del sistema financiero (bancos, casas de bolsa y otros intermediarios financieros) causa al crecimiento económico, siendo solo el crecimiento del IPC el que tiene un impacto significativo en el crecimiento del PIB real. Esto indica que se debe profundizar el sistema financiero, en lo que respecta a la banca y a las entidades de ahorro y crédito popular. Asimismo, se ha mostrado que la evidencia apunta a que la estabilidad de precios y la estabilidad macroeconómica no están en contraposición con el crecimiento económico.

\section{CONCLUSIONES}

El sistema financiero constituye un eslabón crucial entre el ahorro y la inversión; es por ello que su importancia en el crecimiento económico es fundamental. En otras palabras, el volumen y la calidad de la inversión son un determinante importante en el crecimiento económico. La presente investigación mostró cómo el sistema financiero impacta en el crecimiento económico, a través de la estimación de un modelo VAR (2), por lo cual son fundamentales las reformas introducidas desde inicios del 2000 al sistema financiero, ya que se requiere un incremento de la eficiencia del sistema financiero mexicano para canalizar recursos al sector productivo de la economía, porque se observó que la razón IAY es un fuerte motor de crecimiento económico. Esta investigación se encuentra en línea con los resultados encontrados por Bekaert, Harvey y Lundblad (2005), quienes muestran que el sistema financiero incrementa el crecimiento económico porque mejora la asignación de los recursos y, con ello, la tasa de inversión.

Aunado a lo anterior, se observa que México está avanzando con el tema de las reformas estructurales, impulsadas en el 2013, puesse considera que tendrán un impacto importante en la tasa de crecimiento del PIB real, si se aprueban las reformas secundarias y entran en operación. Se espera que en el mediano plazo se observen los beneficios de estas en varios sectores. 
La contribución del trabajo se da en dos sentidos: en el primero estimó el impacto de variables que representaron al sector financiero, y en el segundo aportó evidencia a la literatura existente sobre la controversia de la causalidad entre el sistema financiero y el crecimiento económico, (véanse los resultados analizados de la causalidad en el sentido de Granger). En línea con los hechos estilizados, una economía con un sistema financiero profundo tiene mayores posibilidades de ahorro y con ello de crecimiento, lo cual se mostró con base en la evidencia empírica, para el caso de la economía mexicana en el período 1994:01-2013:04.

En los próximos años se requiere promover la competencia entre el sistema bancario y otros intermediarios financieros para la asignación eficiente de los recursos financieros hacia los sectores productivos, porque si bien causan a la tasa de crecimiento del PIB real, con base en las estimaciones, no se observa un impacto estadísticamente significativo en la muestra estudiada.

La conclusión del presente artículo versa en el hecho de que la estabilidad de precios y la estabilidad macroeconómica no están en contraposición con el crecimiento económico. Como se observó, si se descuida la tasa de crecimiento del INPC podría repercutir en los recursos totales de las entidades de ahorro y crédito popular (prestatarios con menor poder de mercado respecto de los que invierten en la bolsa). Es importante cuidar la estabilidad de precios, la macroeconómica y la financiera, porque la experiencia internacional muestra que todos los excesos (deflaciones o hiperinflaciones) perjudican el buen funcionamiento del sistema económico. Por ejemplo, en las economías con episodios de inflación extrema (hiperinflación) como Argentina, Bolivia, Brasil, Chile, Nicaragua y Perú no les fue fácil recuperarse e impulsar el crecimiento de su economía, mientras que en otro grupo de países con inflación moderada como Colombia, México, Uruguay y Venezuela, la recuperación tuvo un ritmo aceptable.

En este sentido se sabe que la inflación es perjudicial porque distorsiona los precios relativos, afecta los proyectos de inversión (por la incertidumbre), quebranta la información, entre otros factores negativos para el crecimiento. Por estas razones, el Banco de México tiene plena responsabilidad para enfocarse y lograr su objetivo prioritario: garantizar el poder adquisitivo de la moneda.

Se sugiere mantener el papel del Estado en la estimulación del ahorro para invertir en sectores estratégicos y así asegurar el financiamiento del sector productivo. Aunque, el Estado no debe ser proteccionista, no se debe dejar toda la responsabilidad al mercado, porque si bien es cierto que en condiciones de información completa y de no existencia de externalidades, este funciona de manera eficiente. En economías con información asimétrica, externalidades y competencia imperfecta, el Estado 
Influencia de la estabilidad de precios y el desempeño del sistema financiero en el crecimiento económico de México, 1994-2013

debe generar las condiciones para que el buen desempeño de los mercados genere asignaciones eficientes de los recursos.

En resumen, se deben aplicar medidas de política económica armónicas que promuevan el buen funcionamiento de la economía con estabilidad de precios. Además, se debe contar con una adecuada regulación del sistema financiero en pro del crecimiento del país, porque la historia económica muestra cómo sin una regulación financiera apropiada la economía de un país se vulnera. Así, con un sistema financiero regulado y eficiente se disminuiría el riesgo e incentivaría a los agentes económicos a invertir en proyectos productivos, lo cual permitiría el crecimiento económico al aprovechar el desarrollo de economías de alcance y de escala, hecho que sin duda, requiere la economía mexicana.

\section{BIBLIOGRAFÍA}

Akerlof, G. A. (1970). The market for 'lemons': quality uncertainty and the market mechanism. En: The Quarterly Journal of Economics. Agosto, Vol. 84, N. ${ }^{\circ}$ 3, pp. 488-500.

Arestis, P., y Demetriades, P. (1997). Financial development and economic growth: Assessing the evidence. En: The Economic Journal, Vol. 107, N. ${ }^{\circ} 442$, pp. 783-799.

Barro, R. J. (1996). Determinants of economic growth: a cross-country empirical study. En: NBER Working Paper, N. ${ }^{\circ}$ 5698, Cambridge, National Bureau of Economic Research, Agosto, $118 \mathrm{pp}$.

Bekaert, G., Harvey, C. R., y Lundblad, C. (2005). Does financial liberalization spur growth? En: Journal of Financial economics, Vol. 77, ‥ ${ }^{\circ}$ 1, pp. 3-55.

Beck, T. y Levine, R. (2002). Industry growth and capital allocation: does having a marketor bank-based system matter? En: Journal of Financial Economics, Vol. 64 N.․ 2, pp. 147-180.

Beck, T., Levine, R., y Demirgüç-Kunt, A. (2009). Financial institutions and markets across countries and over time: Data and analysis. En: Policy Research Working Paper, N. ${ }^{\circ}$ 4943, The World Bank, Development Reserch Group, Financial and Private Sector Team, mayo, $46 \mathrm{pp}$.

Bresser-Pereira, L. C. (2007). El nuevo desarrollismo y la ortodoxia convencional. En: Economía, UNAM, Vol. 4, N. ${ }^{\circ}$ 10, pp. 7-29.

Caballero, R. J., Farhi, E., y Gourinchas, P. O. (2008). Financial crash, commodity prices and global imbalances. En: NBER Working Paper, N.․ 14521, Cambridge, National Bureau of Economic Research, diciembre, $61 \mathrm{pp}$. 
Carvajal, A., y Zuleta, H. (1997). Desarrollo del sistema financiero y crecimiento económico. En: Borradores de economía, N. ${ }^{\circ}$ 67, Banco de la República, Colombia, 62 pp.

Chari, V. V. (1998). Nobel laureate Robert E. Lucas, Jr.: Architect of modern macroeconomics. En: Journal of Economic Perspectives, Vol. 12, N. ${ }^{\circ}$ 1, pp. 171-186.

Chari, V. V., Kehoe, P. J., y McGrattan, E. R. (2002). Accounting for the great depression. En: American Economic Review, Vol. 92, N. ${ }^{\circ}$ 2, pp. 22-27.

Christopoulos, D. K., y Tsionas, E. G. (2004). Financial development and economic growth: evidence from panel unit root and cointegration tests. En: Journal of development Economics, Vol. 73, N. ${ }^{\circ}$ 1, pp. 55-74.

Demirgüç-Kunt, A., y Maksimovic, V. (2002). Funding growth in bank-based and market-based financial systems: evidence from firm-level data. En: Journal of Financial Economics, Vol. 65, N. ${ }^{\circ}$ 3, pp. 337-363.

Demirgüç-Kunt, A., y Levine, R. (Eds.). (2004). Financial structure and economic growth: A cross-country comparison of banks, markets, and development. Cambridge, MIT press, Agosto, $444 \mathrm{pp}$.

De Gregorio, J., y Guidotti, P. E. (1995). Financial development and economic growth. En: World development, Vol. 23, N. ${ }^{\circ}$, pp. 433-448.

De Gregorio, J., y Lee, J. W. (2004). Growth and Adjustment in East Asia and Latin America. En: Economía, Vol. 5, N. ${ }^{\circ}$ 1, pp. 69-134.

Golosov, M., y Lucas, R. E. (2003). Menu costs and Phillips curves. En: NBER Working Paper, No. 10187, Cambridge, National Bureau of Economic Research, diciembre, 57 pp.

Jung, W. S. (1986). Financial development and economic growth: international evidence. En: Economic Development and cultural change, Vol. 34, N. ${ }^{\circ}$ 2, pp. 333-346.

King, R. G., y Levine, R. (1993). Finance and growth: Schumpeter might be right. En: The quarterly journal of economics, Vol. 108, N. ${ }^{\circ}$ 3, pp. 717-737.

Kregel, J. (2008). Minsky's Cushions of Safety, Systemic Risk and the Crisis in the Subprime Mortgage Market. En: Finance E Bien Commun, Vol. 2-3 N. ${ }^{\circ} 31-32$, pp. 51-59.

Levine, R. (1997). Financial development and economic growth: views and agenda. En: Journal of economic literature, Vol. 35, pp. 688-726.

Levine, R. (2002). Bank-based or market-based financial systems: which is better? En: Journal of financial intermediation, Vol. 11, N. ${ }^{\circ}$, pp. 398 428.

Minsky, H. (1982), The Financial-instability hypothesis: capitalist processes and the behaviour of the economy, En: Kindleberger, Ch. and Jean-Pierre Laffargue (ed.) Financial Crises: Theory, History, and Policy, Cambridge University Press. 301 pp. 
Influencia de la estabilidad de precios y el desempeño del sistema financiero en el crecimiento económico de México, 1994-2013

Romer, D. (2000). Keynesian macroeconomics without the LM curve. En: NBER Working Paper, N. ${ }^{\circ}$ 7461, Cambridge, National Bureau of Economic Research, enero, 43 pp.

Solow, R. M. (2000). Toward a macroeconomics of the medium run. En: The Journal of Economic Perspectives, Vol. 14, N. ${ }^{\circ}$ 1, pp. 151-158.

Summers, L. H. (2008), International Financial Crises: Causes, Prevention, and Cures. En: The American Economic Review, Vol. 90, N. ${ }^{\circ}$, pp. 1-16.

Taylor, J. B. (2000). Teaching modern macroeconomics at the principles level. En: American Economic Review, Vol. 90, N.², pp. 90-94.

Whalen, C. J. (2007). The US credit crunch of 2007: A Minsky moment. En: Public policy brief, N. ${ }^{\circ}$ 92, Jerome Levy Economics Institute of Bard College, octubre, $26 \mathrm{pp}$.

Wray, L. R. (2002). What happened to goldilocks? A Minskian framework. En: Journal of Economic Issues, Vol. 36, N. ${ }^{\circ}$ 2, pp. 383-391.

Wray, L. R. (2008). Financial markets meltdown: what can we learn from Minsky? En: Public policy brief, N. 94A, Jerome Levy Economics Institute of Bard College, octubre, 12 pp. 


\section{ANEXOS}

Anexo A. Gráficas de las series en niveles

PIB real

(Moneda nacional)

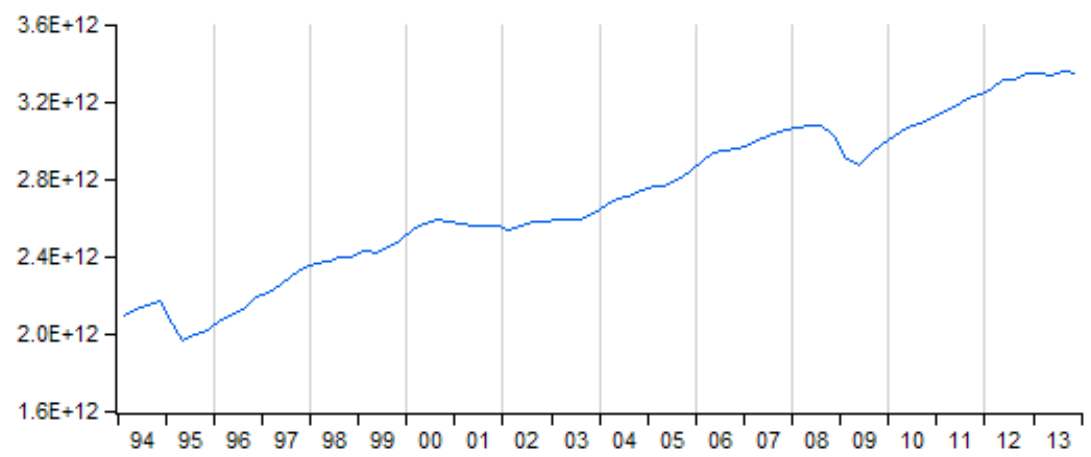

Fuente: elaborada con datos de la OCDE

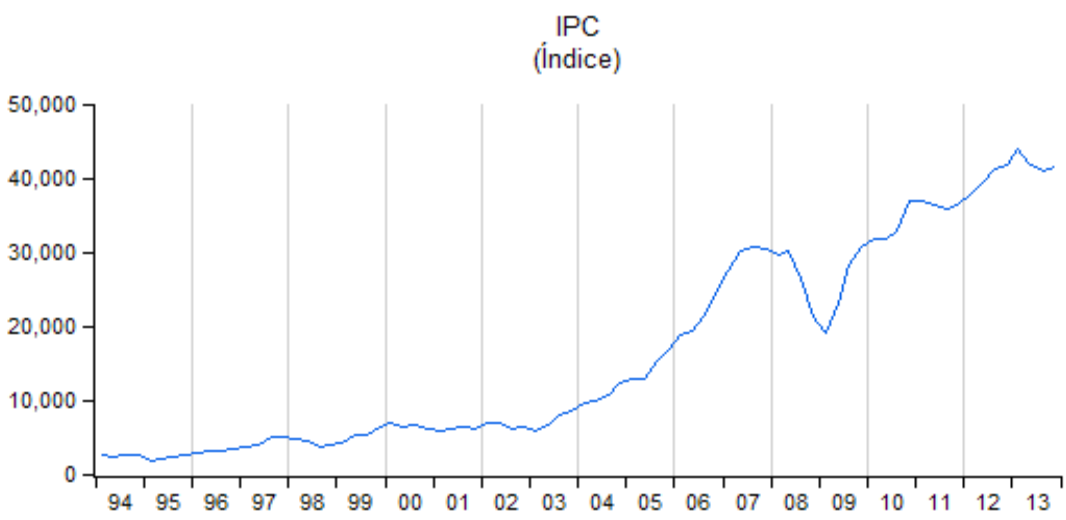

Fuente: elaborada con datos de Banxico 
Financiamiento total otorgado por

la Banca Comercial al Sector no Bancario

(Miles de pesos)

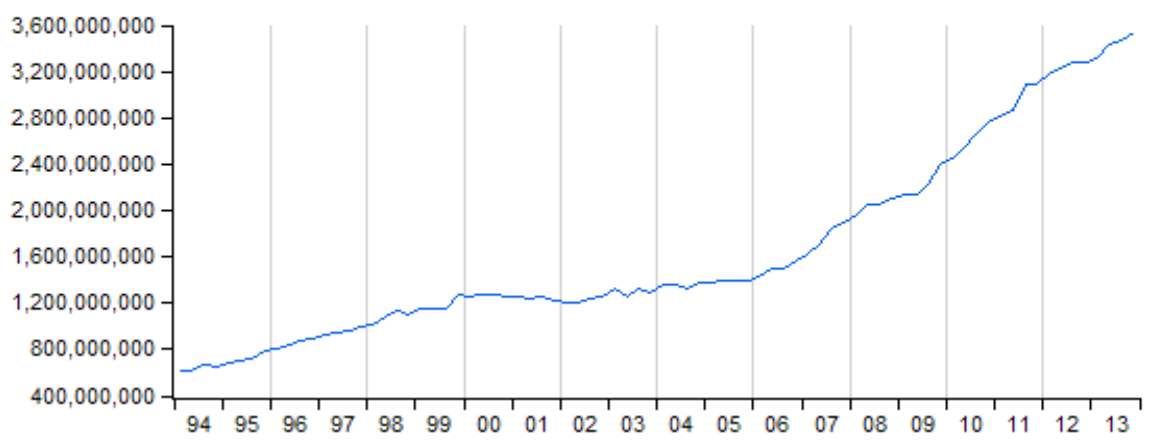

Fuente: elaborada con datos de Banxico

\section{Recursos Totales de las Entidades de Ahorro y Crédito Popular} (Miles de pesos)

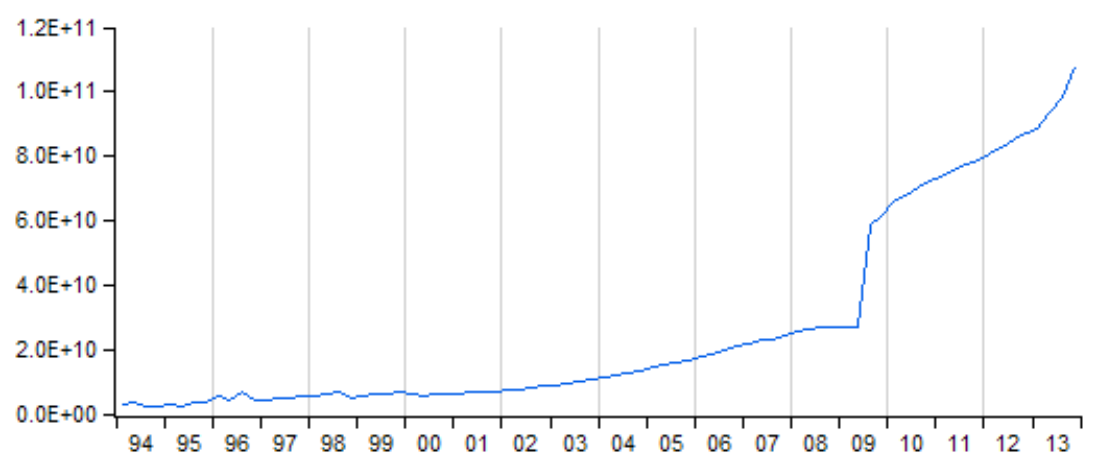

Fuente: elaborada con datos de Banxico

Razón de Inversión a Producto

(Porcentaje)

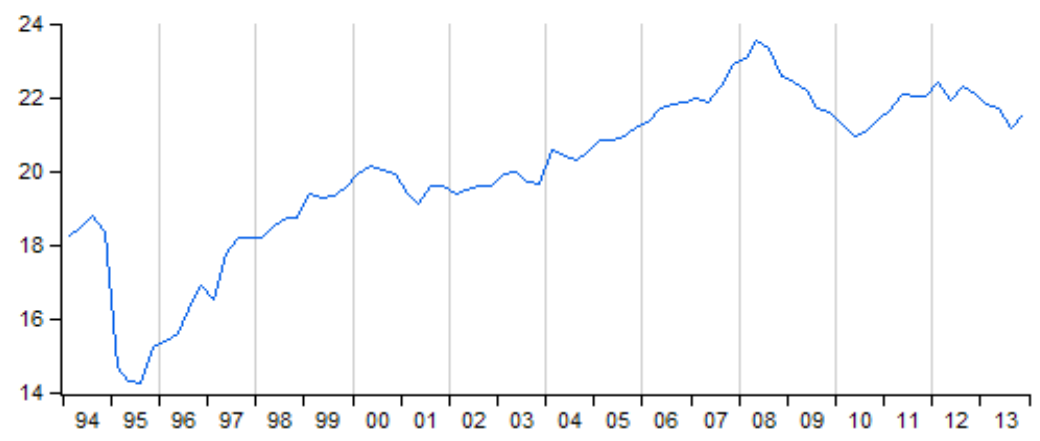

Fuente: elaborada con datos de la OCDE 
INPC

(Índice)

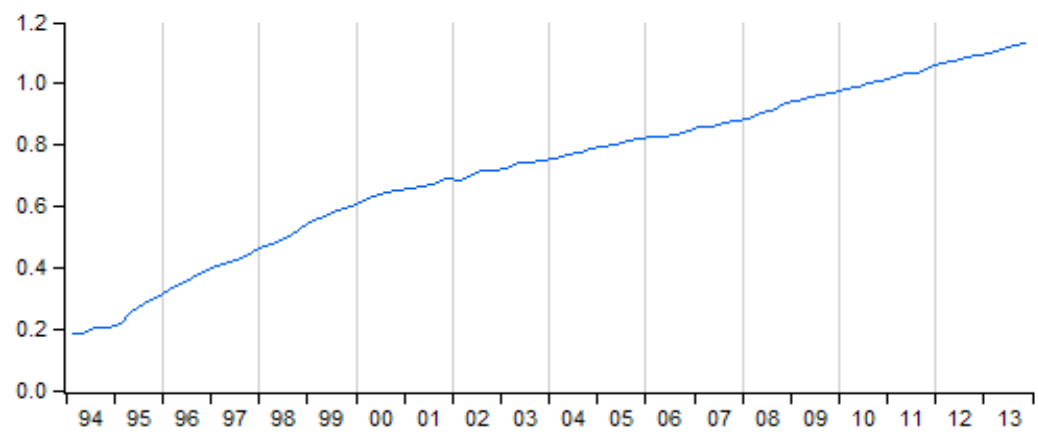

Fuente: elaborada con datos de Banxico

Tasa de rendimiento promedio, CETES 28 días

(Porcentaje)

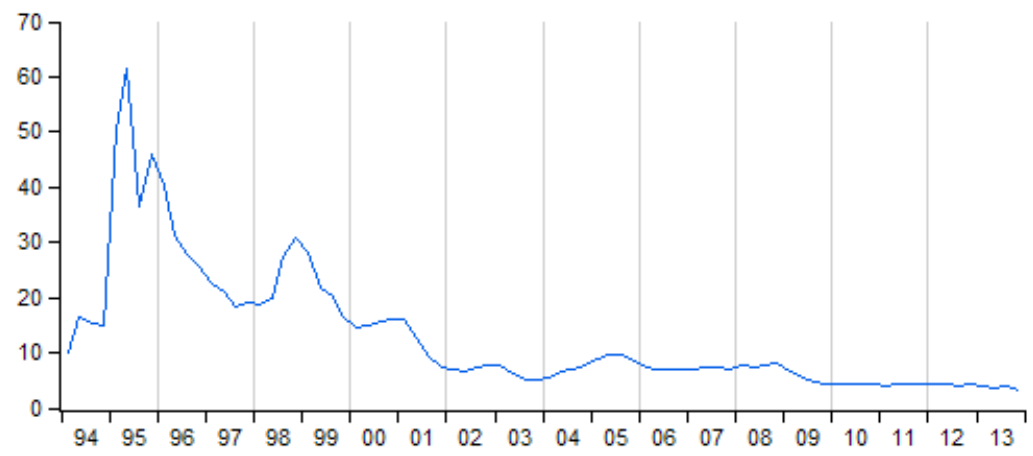

Fuente: elaborada con datos de Banxico

Índice de tipo de cambio real

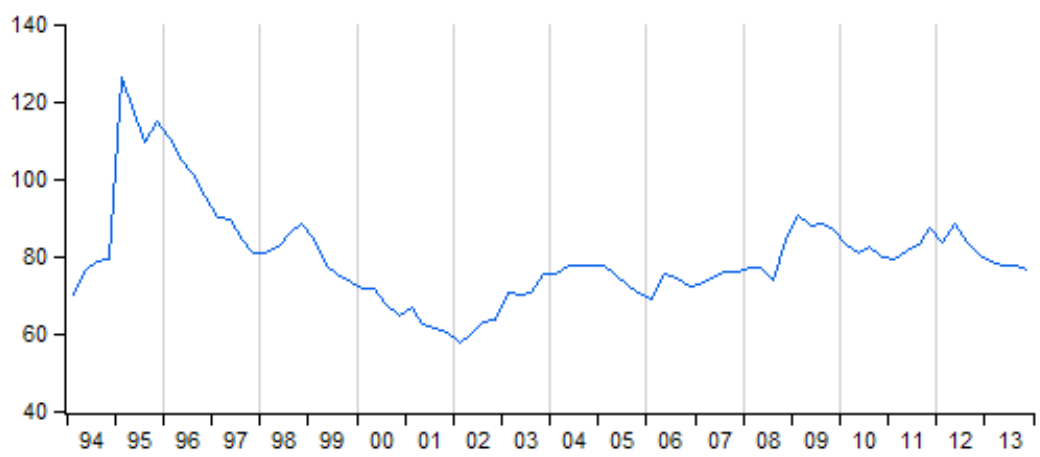

Fuente: elaborada con datos de Banxico 


\section{Anexo B. Especificación del modelo VAR (2)}

$\operatorname{DLY}=\mathrm{C}(1,1) * \operatorname{DLY}(-1)+\mathrm{C}(1,2) * \operatorname{DLY}(-2)+\mathrm{C}(1,3) * \operatorname{DLIAY}(-1)+\mathrm{C}(1,4) * \operatorname{DLIAY}(-2)+$ $\mathrm{C}(1,5) *$ DLIPC $(-1)+\mathrm{C}(1,6) *$ DLIPC $(-2)+\mathrm{C}(1,7)^{*}$ DLFIN _ BC(-1) + C $(1,8)^{*}$ DLFIN _ BC(-2) $+\mathrm{C}(1,9)^{*}$ DLESYCRPOP_RECTOT(-1) + C(1,10)*DLESYCRPOP _ RECTOT $(-2)+\mathrm{C}(1,11)$ $+\mathrm{C}(1,12)^{*} \mathrm{R}+\mathrm{C}(1,13)^{*} \overline{\mathrm{DLINPC}}+\mathrm{C}(1,14)^{*}$ DLTCR

DLIAY $=\mathrm{C}(2,1) * \operatorname{DLY}(-1)+\mathrm{C}(2,2) * \operatorname{DLY}(-2)+\mathrm{C}(2,3) * \operatorname{DLIAY}(-1)+\mathrm{C}(2,4) * \operatorname{DLIAY}(-2)+$ $\mathrm{C}(2,5) *$ DLIPC $(-1)+\mathrm{C}(2,6) * \mathrm{DLIPC}(-2)+\mathrm{C}(2,7) *$ DLFIN _ BC(-1) + C(2,8)*DLFIN _BC(-2) $+\mathrm{C}(2,9) *$ DLESYCRPOP _ RECTOT(-1) + C(2,10)*DLESYCRPOP _ RECTOT(-2) + C $(2,11)$ $+\mathrm{C}(2,12)^{*} \mathrm{R}+\mathrm{C}(2,13)^{*} \mathrm{DLINPC}+\mathrm{C}(2,14)^{*}$ DLTCR

$\operatorname{DLIPC}=\mathrm{C}(3,1) * \operatorname{DLY}(-1)+\mathrm{C}(3,2) * \operatorname{DLY}(-2)+\mathrm{C}(3,3) * \operatorname{DLIAY}(-1)+\mathrm{C}(3,4) * \operatorname{DLIAY}(-2)+$ $\mathrm{C}(3,5) * \mathrm{DLIPC}(-1)+\mathrm{C}(3,6) * \mathrm{DLIPC}(-2)+\mathrm{C}(3,7) *$ DLFIN _ BC(-1) + C(3,8)*DLFIN _ BC(-2) $+\mathrm{C}(3,9) *$ DLESYCRPOP _ RECTOT(-1) + C(3,10)*DLESYCRPOP _ RECTOT $(-2)+\mathrm{C}(3,11)$ $+\mathrm{C}(3,12)^{*} \mathrm{R}+\mathrm{C}(3,13)^{*} \mathrm{DLINPC}+\mathrm{C}(3,14)^{*}$ DLTCR

DLFIN _ BC $=C(4,1) * \operatorname{DLY}(-1)+C(4,2) * \operatorname{DLY}(-2)+C(4,3) * \operatorname{DLIAY}(-1)+\mathrm{C}(4,4) * \operatorname{DLIAY}(-2)+$ $\mathrm{C}(4,5) *$ DLIPC $(-1)+\mathrm{C}(4,6) * \operatorname{DLIPC}(-2)+\mathrm{C}(4,7) *$ DLFIN _BC(-1) + C(4,8)*DLFIN _BC(-2) $+\mathrm{C}(4,9)^{*}$ DLESYCRPOP _ RECTOT(-1) + C(4,10)*DLESYCRPOP _ RECTOT(-2) + C(4,11) $+\mathrm{C}(4,12)^{*} \mathrm{R}+\mathrm{C}(4,13) * \overline{\mathrm{DLINPC}}+\mathrm{C}(4,14) *$ DLTCR

DLESYCRPOP _ RECTOT $=\mathrm{C}(5,1) * \operatorname{DLY}(-1)+\mathrm{C}(5,2) * \operatorname{DLY}(-2)+\mathrm{C}(5,3) * \operatorname{DLIAY}(-1)+$ $\mathrm{C}(5,4) * \operatorname{DLIAY}(-2)+\mathrm{C}(5,5) * \operatorname{DLIPC}(-1)+\mathrm{C}(5,6) * \operatorname{DLIPC}(-2)+\mathrm{C}(5,7) * \operatorname{DLFIN}+\mathrm{BC}(-1)+$ $\mathrm{C}(5,8) *$ DLFIN _ BC(-2) + C(5,9)*DLESYCRPOP _ RECTOT $(-1)+C(5,10) *$ DLESYCRPOP _ RECTOT $(-2)+C(5,11)+C(5,12) * R+C(5,13) *$ DLINPC + C $(5,14) *$ DLTCR 
